\title{
Four Important Blocks of The Last Decade
}

J Balavenkatasubramanian ${ }^{1}$, Gurumoorthi Palanichamy ${ }^{1}$, Senthil Kumar Balasubramanian ${ }^{1}$, Madhanmohan Chandramohan ${ }^{1}$, Vinoth Kumar Subramanian ${ }^{1}$, Satish Raja Selvam Parameswaran ${ }^{1}$

${ }^{1}$ Department of Anaesthesia, Ganga Hospital, Coimbatore, Tamil Nadu, India.

\section{Introduction}

Ultrasonography (USG) guided regional anaesthesia has become the standard practice due to its improved success rate and decreased complications. With the advent of USG regional anaesthesia has flourished in a way that every surgery can be supplemented with a regional block as a part of multimodal analgesia. USG guided regional anaesthesia became rampant in the last decade with many newer inter-fascial plane blocks involving the paraspinal, chest wall and abdominal wall being introduced. Also new approaches for the plexus blocks are being established with improved safety and success. Here in this educational article, we are discussing the intricacies of the USG guided Costoclavicular approach of brachial plexus block, PEricapsular Nerve Group (PENG) block, Serratus Plane block (SPB) and Supra inguinal Fascia Iliaca Block (SIFICB). The costoclavicular approach for brachial plexus block is phrenic nerve sparing and still consistently block all the nerves arising from the cords, making it a safer option in certain population of patient with respiratory compromise. PENG block was introduced in the last decade for providing analgesia for the hip orthopedic procedures and advantage of this block is that there is no motor involvement without any major complications. The SPB is a one of chest wall block used for providing analgesia to the anterolateral chest especially for breast surgery and pain management in rib fractures. Being superficial and technically easier using USG, SPB has been included in the standard pain management for chest trauma. USG guided SIFICB introduced was introduced in 2011 as a novel approach to block the major nerves of lumbar plexus anteriorly but it gained popularity in the recent past for its ability to block femoral nerve, lateral femoral cutanoues nerve and possibly obturator nerve. With this block the above mentioned nerves can be blocked easily without any complications of classical approach for lumbar plexus block.

Address of Correspondence: Dr. Gurumoorthi Palanichamy, Ganga Hospital, Coimbatore, Tamil Nadu, India.

E-mail:drpgurumoorthi@gmail.com

DOI: 10.13107/ijra.2021.v02i01.027 | (C) 2021 International Journal of Regional Anaesthesia | Available on www.ijrajournal.com | This is an Open Access article distributed under the terms of the Creative Commons Attribution Non-Commercial License (http://creativecommons.org/licenses/by-nc/3.0) which permits unrestricted non-commercial use, distribution, and reproduction in any medium, provided the original work is properly cited.

How to cite this article: Balavenkatasubramanian J, Palanichamy G, Balasubramanian S, Chandramohan M, Subramanian VK, Parameswaran SRS | Four Important Blocks of The Last Decade | International Journal of Regional Anaesthesia | January-June 2021; 2(1): 54-62. 


\section{Costoclavicular Brachial Plexus Block- A Phrenic Nerve Sparing Novel Block}

\section{Introduction:}

Costoclavicular brachial plexus block is a new approach for infraclavicular brachial plexus block, targeting the cords of brachial plexus. At the costoclavicular space (CCS), and in contrast to that at the Lateral Infra Clavicular (LIC) space, all the cords of the brachial plexus are clustered together lateral to the first part of axillary artery and share a consistent relation to one another and to the axillary artery. In costoclavicular brachial plexus block, the cords are located superficially when compared to classical infraclavicular brachial plexus block.

\section{Scanning Technique:}

Patient being in supine position with ipsilateral arm abducted to 90 degree and the head turned slightly to the contralateral side of the brachial plexus block. A high frequency linear array transducer $(8-13 \mathrm{MHz})$ is positioned initially above the midpoint of the clavicle in the transverse orientation, with its orientation marker

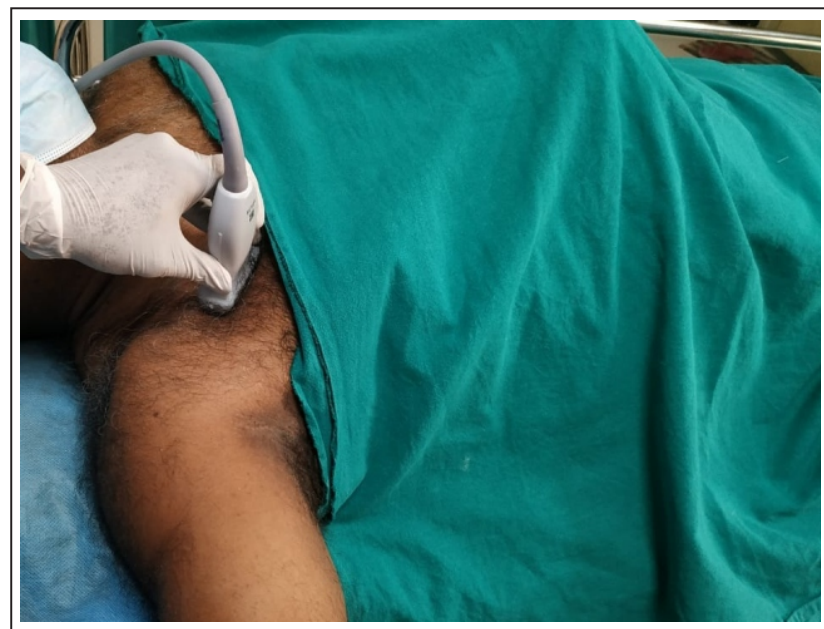

Figure 1: USG Probe Position and with abducted arm

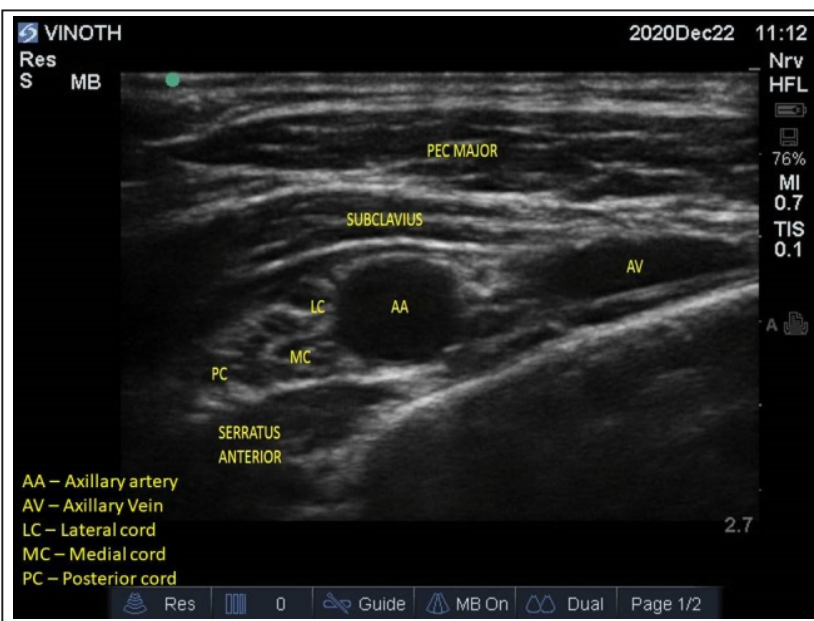

Figure 3 A: An optimized image of costoclavicular space depicts the three cords directed laterally. Then the transducer is gently moved caudally until it slipped off the inferior border of the clavicle and the axillary artery (first part) and vein are visualized. With the transducer being in the same position, a gentle cephalad tilt is given to direct the ultrasound beam towards the CCS, the space between the posterior surface of the clavicle and the second rib [1]. The ultrasound image is optimized, until all three cords of the brachial plexus are clearly visualized lateral to axillary artery (Fig. 1-5).

\section{Needling Technique:}

Under all aseptic precautions, a $22 \mathrm{G}$ echogenic needle is inserted either in-plane (lateral to medial or medial to lateral) or out of plane approach to reach the centre of three cords [2] (Fig. 6 \& 7). Care must be taken to avoid needle insertion with the cephalic vein or thoracoacromial artery in view, as this would indicate needle insertion distal to the CCS. For the same reason, it is imperative that the subclavius muscle is visualized at all times during the needle insertion. A total volume of $20 \mathrm{ml}$ of local anaesthetic is injected in small aliquots and at a single site over 2 to 3 minutes [3].

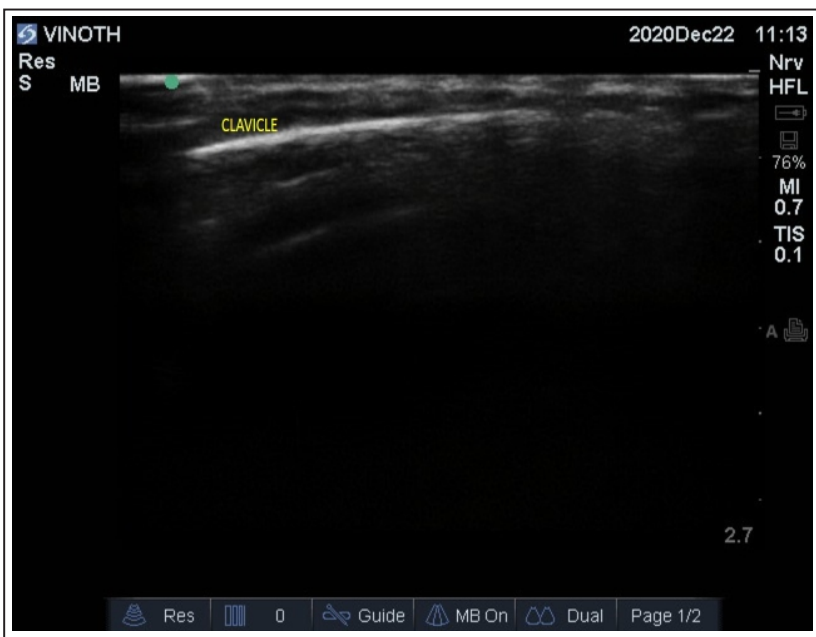

Figure 2: Sonogram with probe over clavicle

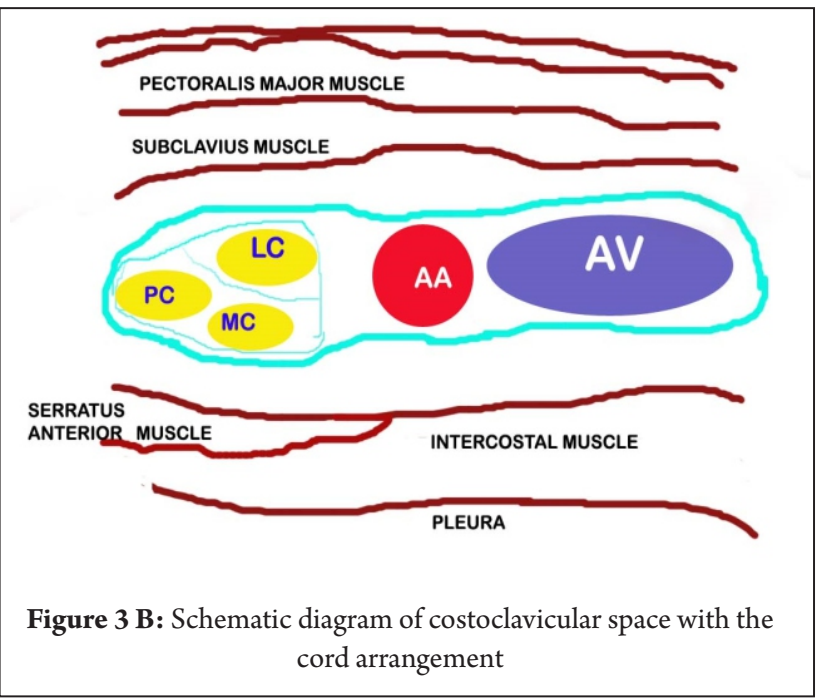




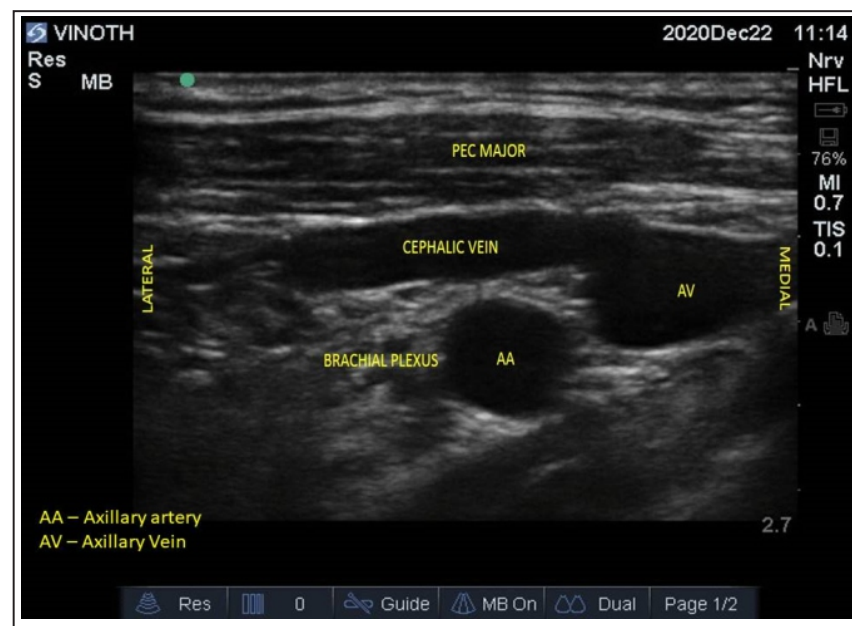

Figure 4: Sonogram showing the cephalic veins arching over cords

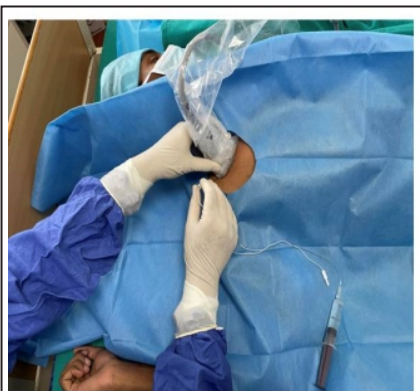

In plane approach

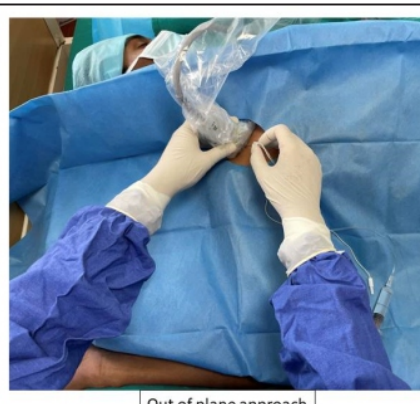

Out of plane approach
Figure 6: $\mathrm{CCB}$ with in-plane and out of plane approach

\section{Drug spread:}

A cadaveric study in Five cadavers demonstrates that ultrasound guided injection with $20 \mathrm{ml}$ of $0.1 \%$ methylene blue in the costoclavicular space spreads cephalad to the brachial plexus in the supraclavicular space, consistently reaching the suprascapular nerve and all the trunks and cords of brachial plexus, while sparing the phrenic nerve [4].

\section{Advantages:}

- The incidence of hemidiaphragmatic paralysis is significantly lower with costoclavicular than with supraclavicular brachial plexus block [5].

- Less incidence of pneumothorax

- The cords are densely packed which can reduce the LA volume/dose [3].

- Ultrasound guided single shot costoclavicular block with low volume of LA can provide reliable and safe anaesthesia for single stage bilateral upper limb surgeries.

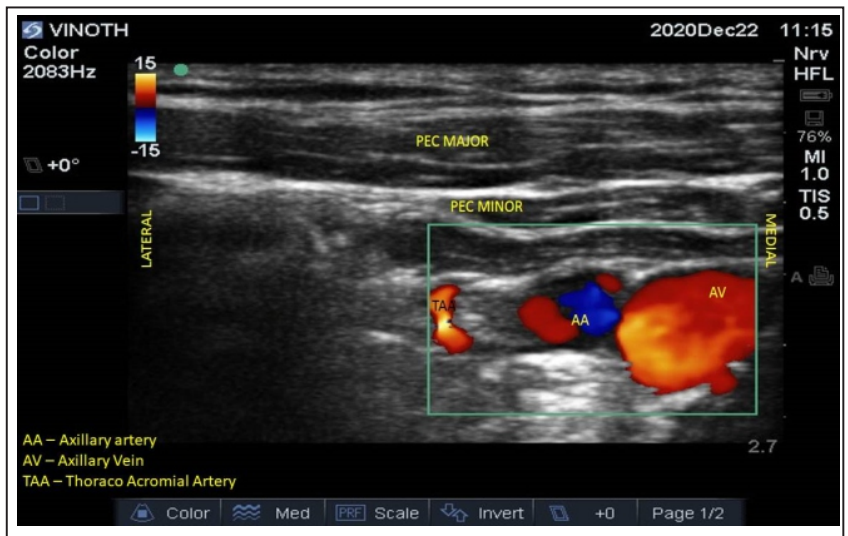

Figure 5: Doppler sonogram showing origin of thoracoacromial artery

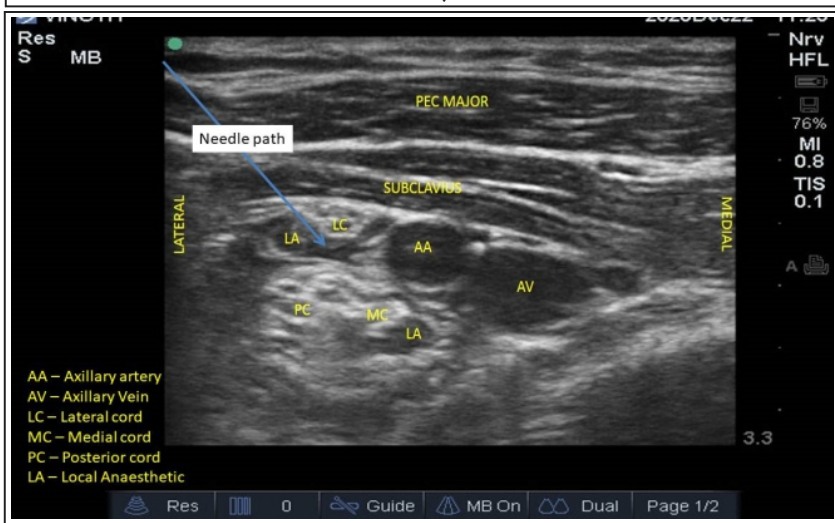

Figure 7: Spread of local anaesthestic

\section{References:}

1. Silva MD. The costoclavicular syndrome: a 'new cause'. Ann Rheum Dis. 1986; 45: 916-20

2. Li JW, Songthamwat B, Samy W, Sala-Blanch X, Karmakar MK. Ultrasound-Guided Costoclavicular Brachial Plexus Block: Sonoanatomy, Technique, and Block Dynamics. Reg Anesth Pain Med.2017 Mar/Apr;42(2):233-240.

3. Wong MH, Karmakar MK, MokLYH, Songthamwat B, Samy W. Minimum effective volume of $0.5 \%$ ropivacaine for ultrasoundguided costoclavicular brachial plexus block: A dose finding study. Eur J Anaesthesiol. 2020 Sep;37(9):780-786. doi: 10.1097/EJA.0000000000001287.PMID: 32740321.

4. Koyyalamudi V, Langley NR, Harbell MW, Kraus MB, Craner RC, Seamans DP. Evaluating the spread of costoclavicular brachial plexus block: an anatomical study. Reg Anesth Pain Med. 2021 Jan;46(1):31-34. doi: 10.1136/rapm-2020-101585. Epub 2020 Oct6. PMID: 33024005.

5. Oh C, Noh C, Eom H, Lee S, Park S, Lee S, Shin YS, Ko Y, Chung W, Hong B. Costoclavicular brachial plexus block reduces hemidiaphragmatic paralysis more than supraclavicular brachial plexus block: retrospective, propensity score matched cohort study. Korean J Pain.2020 Apr 1;33(2):144-152. 


\section{Serratus Plane Block}

\section{Introduction}

Providing adequate perioperative analgesia for chest wall surgeries or rib fractures poses significant challenge to anaesthesiologist everyday. Breast surgeries are associated with persistent postoperative pain in $30-55 \%$ of the patients, mostly progressing to chronic pain. Serratus plane block (SPB) has emerged over the years as a novel technique for analgesia of the anterolateral chest wall $[1]$.

\section{Anatomy}

Chest wall is predominantly innervated by the cutaneous branches of the intercostal nerve. The lateral cutaneous branches of the T3-T9 thoracic intercostal nerves pierces internal intercostal muscle, external intercostal muscle (EIM) and serratus anterior muscle (SAM) to supply lateral chest wall (Fig. 1). Thoracodorsal nerve (TDN) and Long thoracic nerve (LTN) lie in the plane between latissimus dorsi muscle (LDM) and serratus anterior muscle and innervate lateral chest musculature.

Two variations of SPB have been described in the literature [2].

1) Superficial SPB: LA is injected between LDM and SAM.

2) Deep SPB: LA is injected between SAM and rib or EIM. Both superficial and deep SPB provides ipsilateral sensory block in T2-T9 dermatomes. In addition, superficial SPB also blocks LTN and TDN which might lead to intraoperative anatomical distortion and surgical interference. Greater dermatomal spread is expected in deep SPB, because respiratory movements allow LA to disperse along the space due to capillary action [3]. Cadaveric studies with methylene blue dye have demonstrated a spread of
$10 \mathrm{~cm}$ and $13 \mathrm{~cm}$ antero-posterior and cephalo-caudal direction respectively [4].

\section{Indications}

1) Breast surgery, 2) Rib fractures, 3) Thoracoscopy, 4) Thoracotomy, 5) Chronic pain after mastectomy 6) Post herpetic neuralgia

\section{Contraindications}

1) Patient refusal 2) Allergic to local anaesthetics 3) Subcutaneous emphysema 4) Presence of chest tubes 5) Prior thoracotomy or Latissimus dorsi flap surgery

\section{Technique:}

Place the patient in supine position with the abduction of ipsilateral arm (Fig. 2). A high frequency linear ultrasound transducer is placed over the deltopectoral groove and moved inferolaterally in parasagittal oblique plane until the 5th rib, LDM superficially and SAM deeper are visualized (Fig. 3) The Thoracodorsal artery (TDA) runs between these two muscles and helps to delineate the plane between them. A22G $5 \mathrm{~cm}$ short bevel needle is introduced via in-plane technique from anterior to posterior direction in the midaxillary line. In superficial SPB, the needle tip is placed in the fascial plane between two muscles [5] (Fig. 4). Hydrolocalisation helps to delineate the two muscles. In deep SPB, needle tip is placed between SAM and 5th rib. A volume of $0.4 \mathrm{ml} / \mathrm{kg} \mathrm{LA}$ (0.25\% BUPIVACAINE/0.2\% ROPIVACAINE) with Dexamethasone $4 \mathrm{mg}$ provides analgesia for18-24 hour duration. Increasing the volume of LA from 20 to $40 \mathrm{ml}$ will cover to more dermatomes. Continuous catheters [6] can be inserted with LA infusions at $6-8 \mathrm{ml} / \mathrm{hr}$.

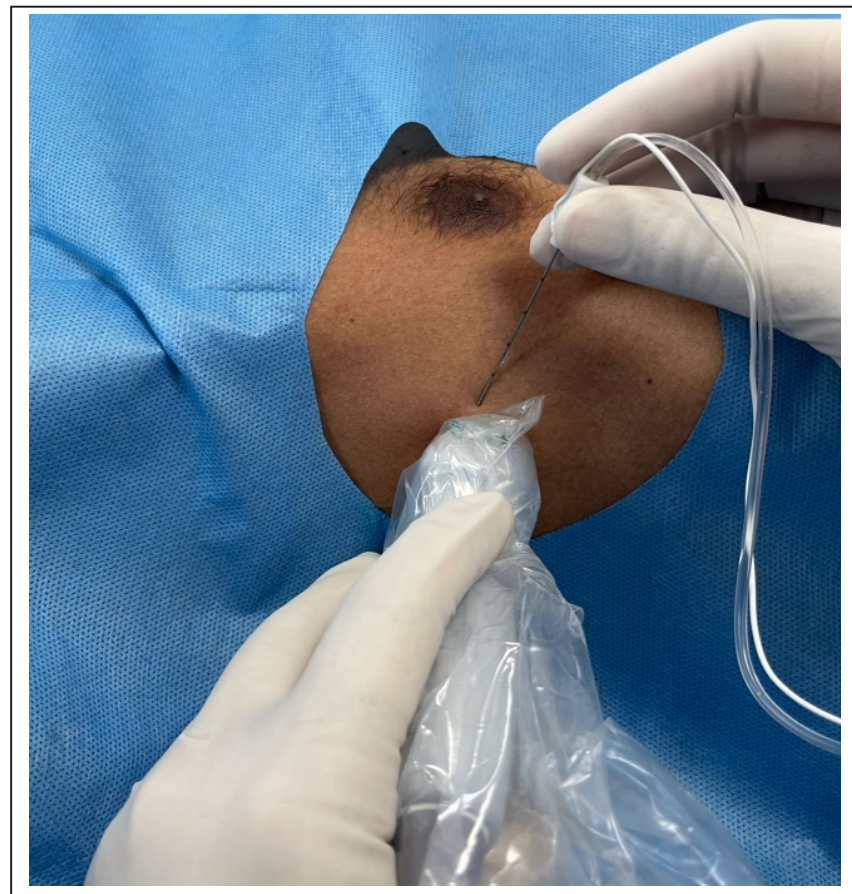

Figure 2: Ultrasound Probe Position For Serratus Plane Block At The Level Of 5th Rib In The Mid-Axillary Line
Figure 1: Cross sectional illustration of intercostal nerv and
Figure 1: Cross sectional illustration of inte
surrounding muscles

Serratus anterior

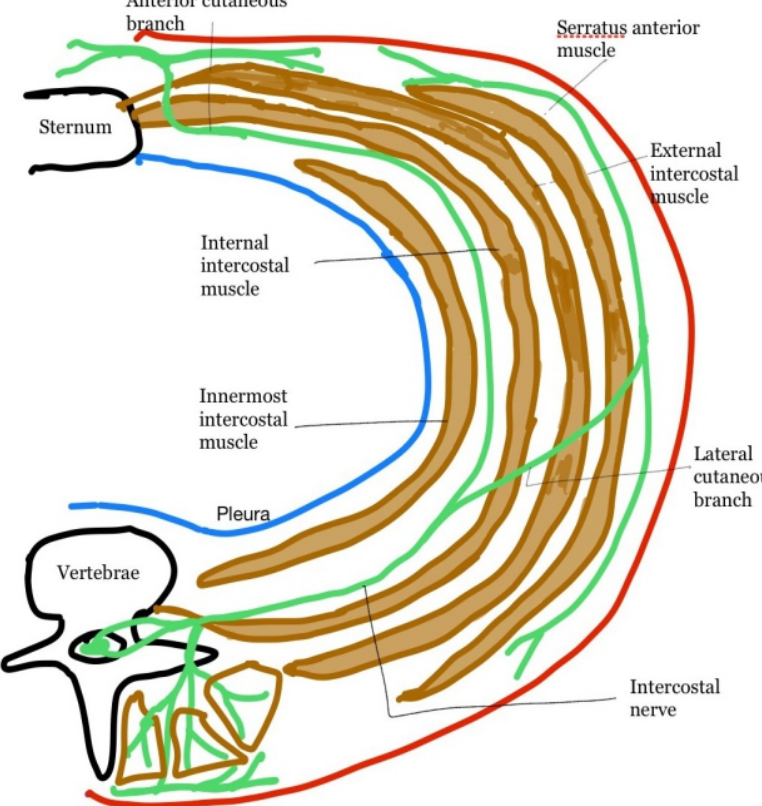




\section{Complications}

1) Local anaesthetic systemic toxicity 2) Vascular injury 3) Pleural puncture

\section{Conclusion:}

Serratus plane block can be used as a novel technique or a component of multimodal analgesia of chest wall in perioperative or trauma care settings with decreased opioid consumption [7].

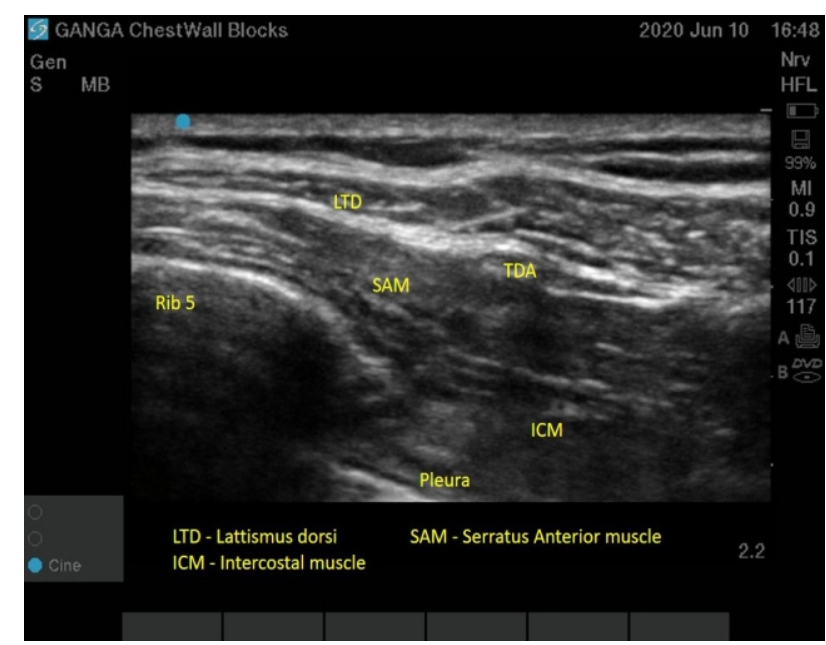

Figure 3: Ultrasound Image Demonstrating Serratus Plane Between Latissimus Dorsi And Serratus Anterior Containing Thoracodorsal Artery At The Level Of $5^{\text {th }}$ Rib;TDA- ThoracodorsalArtery

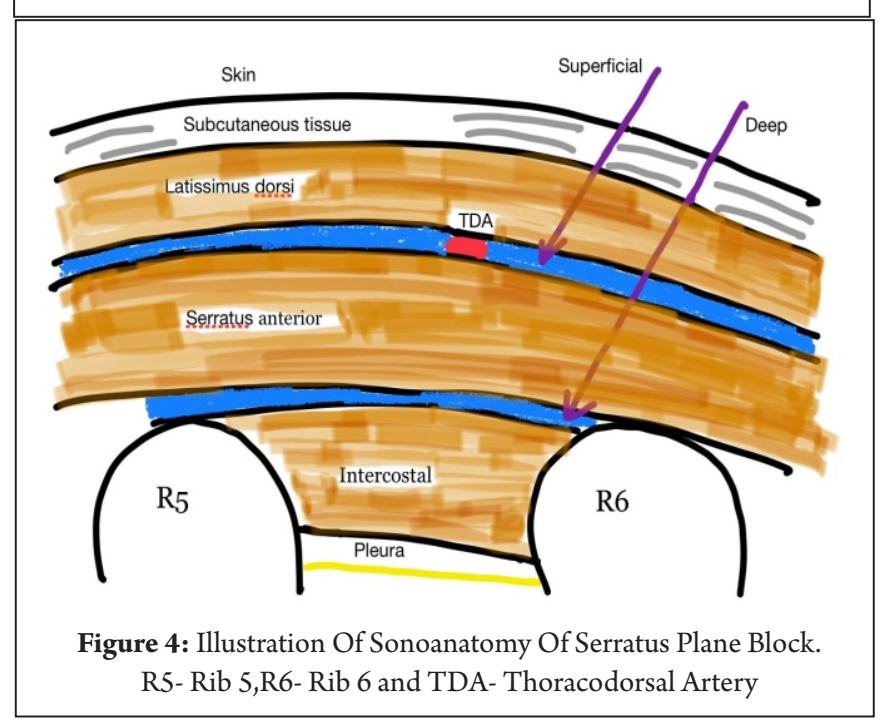

\section{Reference:}

1. Blanco R, Parras T, McDonnell JG, et al. Serratus plane block: a novel ultrasound-guided thoracic wall nerve block. Anaesthesia. 2013;68:1107-1113.

2. Piracha MM, Thorp SL, Puttanniah V, et al. "A tale of two planes": deep versus superficial serratus plane block for postmatectomy pain syndrome. Reg Anesth Pain Med. 2017;42:259-262.

3. Jadon A, Jain P. Serratus Anterior Plane Block-An Analgesic Technique for Multiple Rib Fractures: A Case Series. American J Anesth Clin Res. 2017;3(1):001-004.

4. Mayes J, Davison E, Panahi P, et al. An anatomical evaluation of the serratus anterior plane block. Anaesthesia. 2016;71:10641069 .

5. ChinKJ.Thoracic wall blocks: from paravertebral to retrolaminar to serratus to erector spinae and back again-a review of evidence. Best Pract Res Clin Anaesthesiol. 2019;33:6777.

6. Sayan Nath, Devesh Bhoj, Virender Kumar mohan et al. USGguided continuous erector spinae block as a primary mode of perioperative analgesia in open posterolateral thoracotomy: A report of two cases. SaudiJ Anaesthesia,2018 July-Sep;12(3):471474.

7. Chong M, et al. The serratus plane block for postoperative analgesia in breast and thoracic surgery: a systematic review and meta- analysis Reg Anesth Pain Med 2019;44:1066-1074. doi:10.1136/rapm-2019-100982 


\section{PENG [PEricapsular Nerve Group $]$ Block}

\section{Introduction}

Nociceptive fibers are predominantly concentrated in the anterior hip capsule and the posterior capsule has predominantly mechanoceptors [1]. Anterior capsule is innervated by articular branches from femoral nerve, obturator nerve and accessory obturator nerve [2]. The high articular branches of femoral (FN) and accessory obturator ( $\mathrm{AON}$ ) nerves are found to be consistently present in the musculofascial plane between psoas muscle and the pubic ramus between iliopubic eminence (IPE) and anterior inferior iliac spine (AIIS), and the obturator (ON) nerve along with its articular branches are found a little further medially in the plane between pectineus and obturator externus $[2,3]$. LA deposited in this musculofascial plane would involve articular branches of $\mathrm{FN}$, $\mathrm{AON}$ and spread medially in the subpectineal plane to involve articular branches of $\mathrm{ON}$ [4].

Patient is supine, curvilinear low frequency $(2-5 \mathrm{MHz})$ ultrasound is placed transversely on AIIS and then aligned with pubic ramus by rotating the farther end of the probe towards pubic ramus by approximately 45 degrees (Figure 1). The iliopsoas muscle and tendon would be seen on the pubic ramus, the medial and lateral boundaries being IPE and AIIS. A $10 \mathrm{~cm}, 22 \mathrm{G}$ needle is inserted in an in-plane approach from lateral to medial to bring the tip in the myofascial plane between psoas and pubic ramus (Figure 2). $20 \mathrm{ml}$ of LA deposited here has been found to produce satisfactory analgesia [4] (Figure 3).

PENG block provides pain relief in intra-hip capsule pathologies and procedures, with no associated quadriceps weakness $[4,5,6]$. Patient discomfort due to longer needle path, difficulty visualizing the needle, inadvertent motor weakness, potential for injury to femoral nerve and vessels or the ureter, large abdomen pannus obscuring sonoanatomy are some of the difficulties associated with PENG block $[7,8,9,10]$.

Pericapsular nerve group (PENG) block is a novel regional analgesia technique4. Current evidence as to its efficacy in hip pain is mainly from case reports and case series, where it has been seen to provide sufficient analgesia with minimal reported side effects [11]. PENG block can be considered as an analgesic technique for hip pathologies, that is alternative to lumbar plexus block, femoral nerve block or fascia iliaca blocks, particularly when motor weakness has to be avoided $[11]$.

Figure 3: Image Depicting The Sonogram During Placement Of A Peng Block. AIIS- Anterior Inferior Iliac Spine; IPE- Iliopubic Eminence; Pm- Psoas Tendon; FA- Femoral Artery; L- Lateral; Red Arrow Indicates Needle Path

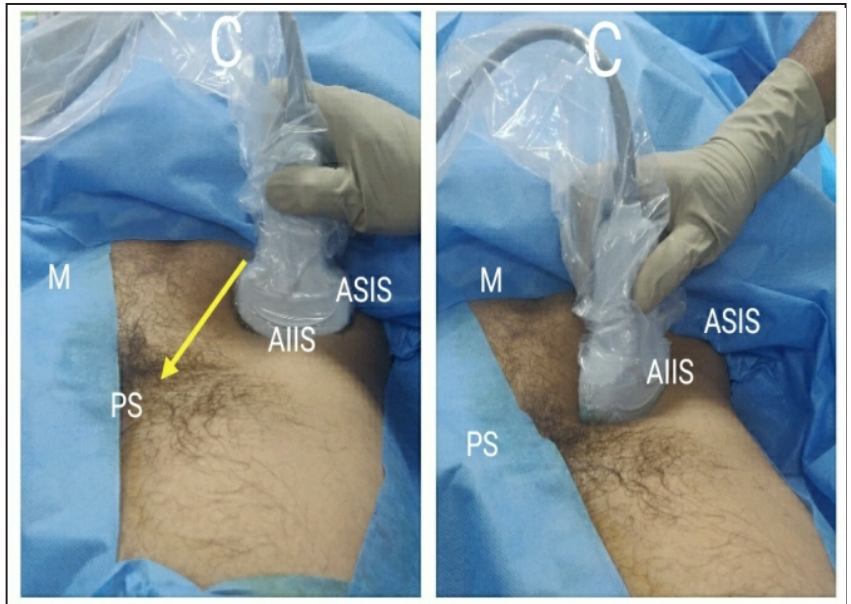

Figure 1: Ultrasound Probe Orientation During Peng Block. Image On The Left Shows Initial Placement Of The Probe Transversely On

The ASIS. Image On The Right Shows Final Position Of The

Ultrasound Probe Aligning With AIIS And PS. ASIS, Anterior Superior Iliac Supine; AIIS Anterior Inferior Iliac Spine; PS- Pubic

Symphysis; M- Medial; C- Cranial End; Yellow Arrow Indicates Rotation Of The Medial End Of The Ultrasound Probe Towards The Pelvis
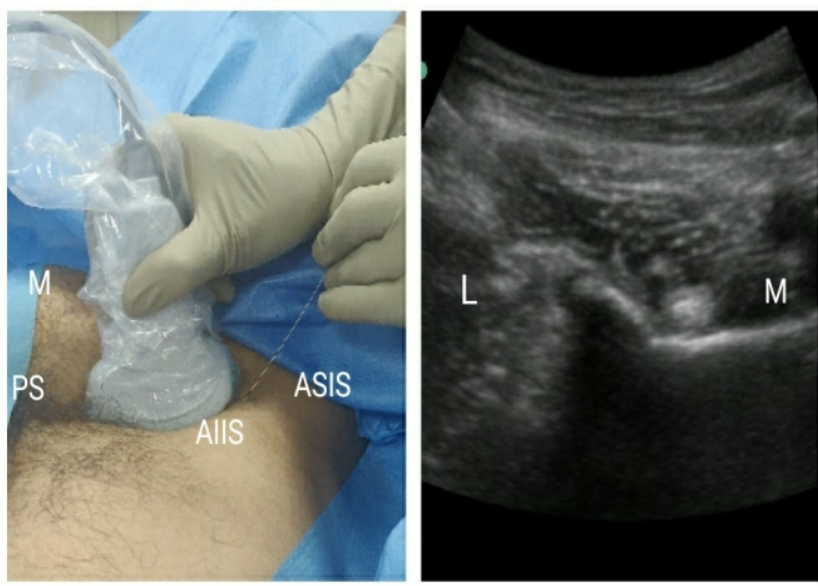

Figure 2: Image On The Left Shows Needle Insertion In An In-Plane, Lateral To Medial Technique During Placement Of Peng Block. Image On The Right Shows Corresponding Sonogram. ASIS, Anterior Superior Iliac Spine; AIIS, Anterior Inferior Iliac Spine; Ps- Pubic Symphysis; M- Medial; L- Lateral

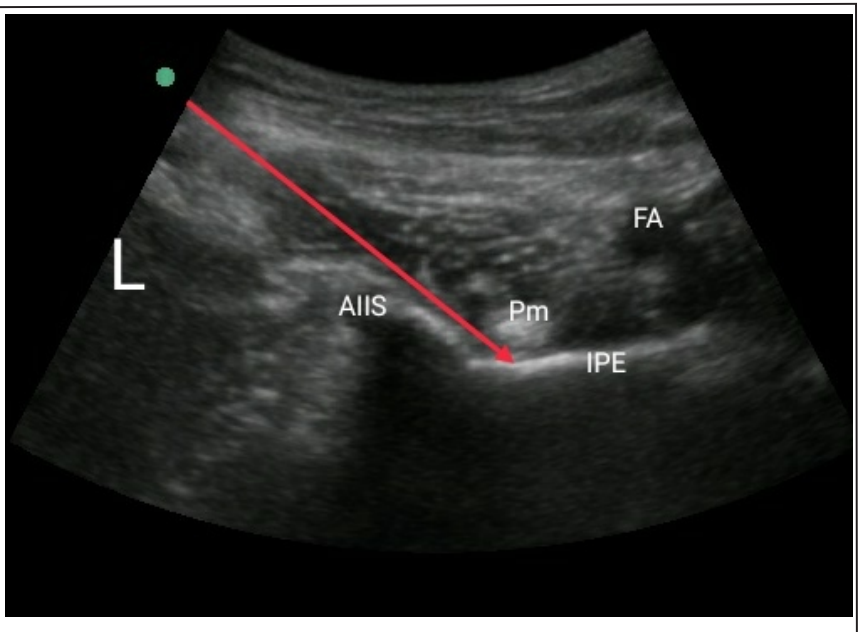




\section{References:}

1. Bugada D, Bellini V, Lorini LF, Mariano ER. Update on Selective Regional Analgesia for Hip Surgery Patients. Anesthesiol Clin.2018;36(3):403-415. doi:10.1016/j.anclin.2018.04.001

2. Short AJ, Barnett JJG, Gofeld M, et al. Anatomic Study of Innervation of the Anterior Hip Capsule: Implication for ImageGuided Intervention. Reg Anesth Pain Med. 2018;43(2):186192. doi:10.1097/AAP.0000000000000701

3. Nielsen TD, Moriggl B, Søballe K, Kolsen-Petersen JA, Børglum J, Bendtsen TF. A cadaveric study of ultrasound-guided subpectineal injectate spread around the obturator nerve and its hip articular branches. Reg Anesth Pain Med. 2017;42(3):357361. doi:10.1097/AAP.0000000000000587

4. Girón-Arango L, Peng PWH, Chin KJ, Brull R, Perlas A. Pericapsular Nerve Group (PENG) Block for Hip Fracture. Reg Anesth Pain Med.2018;43(8):859-863. doi:10.1097/AAP.0000000000000847

5. Bilal B, Öksüz G, Boran ÖF, Topak D, Doğar F. High volume pericapsular nerve group (PENG) block for acetabular fracture surgery: A new horizon for novel block. J Clin Anesth. 2020;62. doi:10.1016/j.jclinane.2020.109702

6. Kukreja P, Avila A, Northern T, Dangle J, Kolli S, Kalagara H. A Retrospective Case Series of Pericapsular Nerve Group (PENG) Block for Primary Versus Revision Total Hip Arthroplasty Analgesia. Cureus. 2020;12(5). doi:10.7759/cureus.8200
7. Acharya U, Lamsal R. Pericapsular Nerve Group Block: An Excellent Option for Analgesia for Positional Pain in Hip Fractures. Case Rep Anesthesiol. 2020;2020:1-3. doi:10.1155/2020/1830136

8. Mistry T, Sonawane KB, Kuppusamy E. PENG block: Points to ponder. Reg Anesth Pain Med. 2019;44(3):423-424. doi:10.1136/rapm-2018-100327

9. Enes Aydin M, Borulu F, Ates I, Kara S, Ahiskalioglu A. Letters to the Editor A Novel Indication of Pericapsular Nerve Group (PENG) Block: Surgical Anesthesia for Vein Ligation and Stripping. J Cardiothorac Vasc Anesth. 2020;34:843-845. doi:10.1053/j.jvca.2019.08.006

10. Yu HC, Moser JJ, Chu AY, Montgomery SH, Brown N, Endersby RVW. Inadvertent quadriceps weakness following the pericapsular nerve group (PENG) block. Reg Anesth Pain Med. 2019;44(5):611-613. doi:10.1136/rapm-2018-100354

11. Morrison C, Brown B, Lin DY, Jaarsma R, Kroon H. Analgesia and anesthesia using the pericapsular nerve group block in hip surgery and hip fracture: A scoping review. Reg Anesth Pain Med. 2021;46(2):169-175. doi:10.1136/rapm-2020-101826 


\section{Suprainguinal Fascia Iliaca Block-Is It The True 3 In 1 Block?}

\section{Introduction}

The fascia iliaca compartment is a potential space lying between the fascia ilaca anteriorly and the iliacus and psoas muscles (iliopsoas) posteriorly. Fascia iliaca block (FICB) is a peripheral compartment block, traditionally given below the inguinal ligament between the fascia iliaca and iliopsoas muscle, thereby blocking the femoral nerve (FN) and sometimes lateral femoral cutaneous nerve (LFCN). Before the advent of ultrasound the block was performed using landmark with "double pop" technique infra inguinally [1] and later by a modified suprainguinal approach [2]. The block was mainly performed for analgesia of the hip surgery. With the introduction of USG to RA, FICB block success rate has increased and it blocks the femoral nerve consistently; whereas the LFCN which is lying superficial to the fascia iliaca and underneath the fascia lata explains the reason behind its sparing in infrainguinal FICB. The branches of the lumbar plexus namely the obturator nerve (ON), FN, LFCN formed in the substance of the psoas muscles and they emerge out of the muscle. FN and LFCN course in the anterior surface of iliopsoas underneath the fascia iliaca till the inguinal ligament level and distal to that they branch and remain in different fascial planes. The LFCN pierces the fascia iliaca at the level of inguinal ligament to lie in the plane between fascia iliaca and fascia lata where it gives sensory branches anterior and lateral divisions supplying the lateral thigh. The common $\mathrm{ON}$ emerges medial to the psoas muscle and lateral to sacroiliac joint before leaving pelvis via obturator canal. The FN and LFCN lie in the fascia iliaca compartment supra inguinally and the $\mathrm{ON}$ runs in the medial side of the psoas muscle. Suprainguinal FICB should block FN and LFCN consistently and possibly ON when adequate volume of local anaesthetic (LA) is injected. Kris Vermeylen et al [3] suggested based on combining CT and dissection findings that the volume to reach the FN, ON, and LFCN with a supra inguinal FICB was $40 \mathrm{ml}$.

Indications: Proximal hip fracture and femur shaft fracture for perioperative analgesia.

Contraindications: Inguinal swellings (lymph nodes, hernia, varicocele), Hernioplasty.

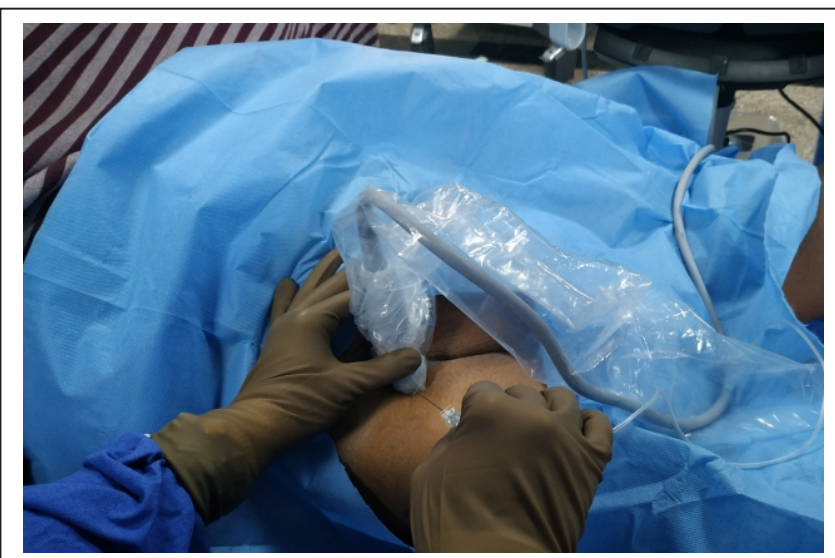

Figure 1: USG Probe Position

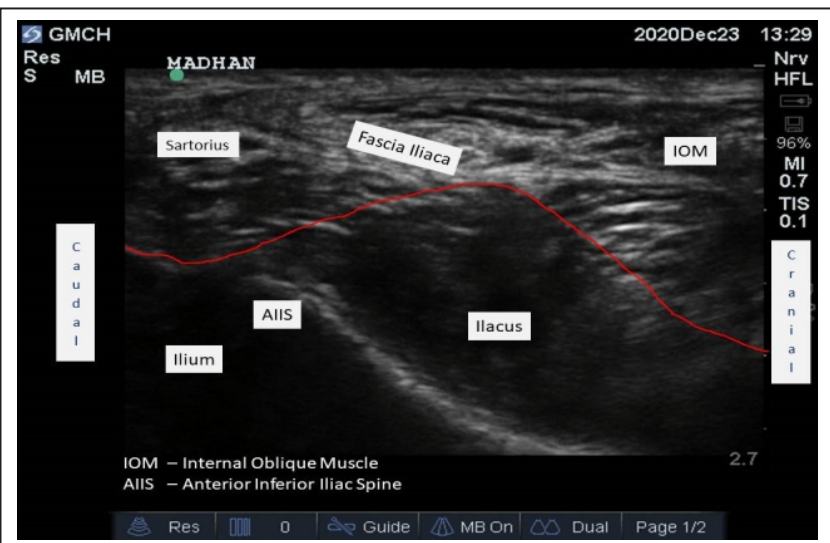

Figure 2: Sonogram Showing The Optimized Image For Suprainguinal FICB

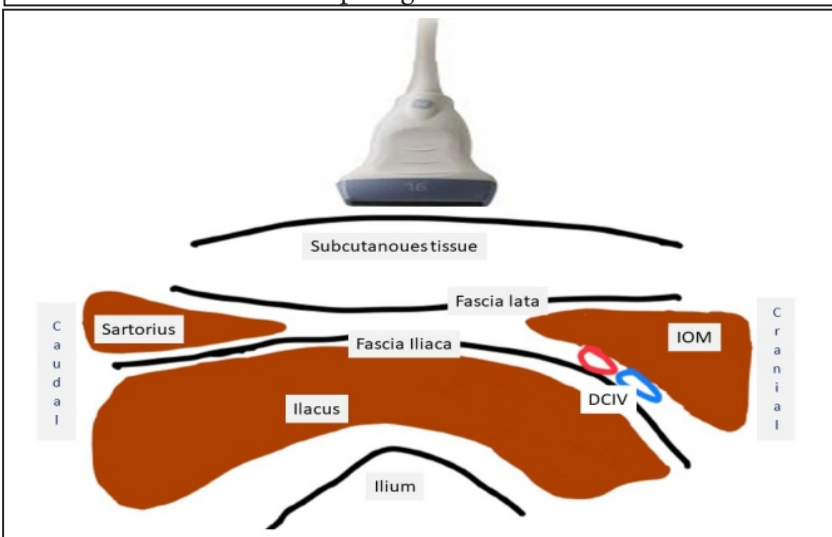

Figure 3: Schematic Diagram Showing The Suprainguinal FICB Anatomy

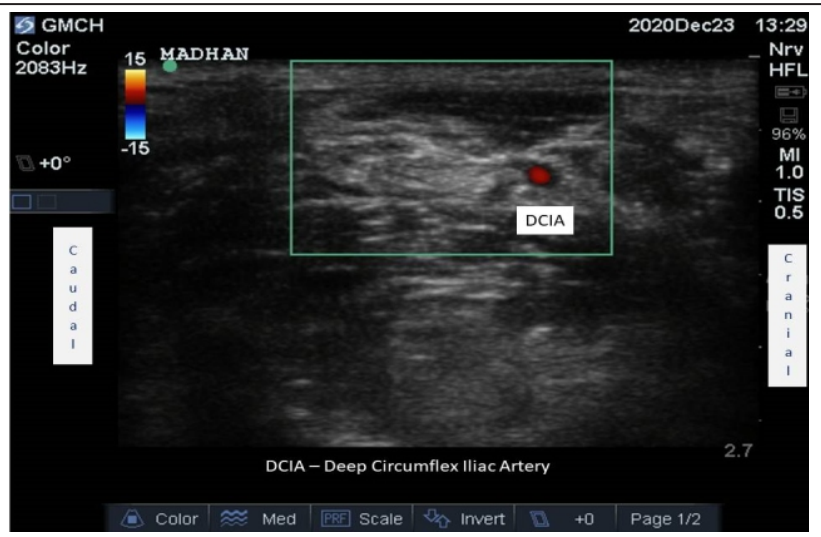

Figure 4: Sonogram Showing Deep Circumflex Iliac Artery Over The Suprainguinal Fascia Iliaca

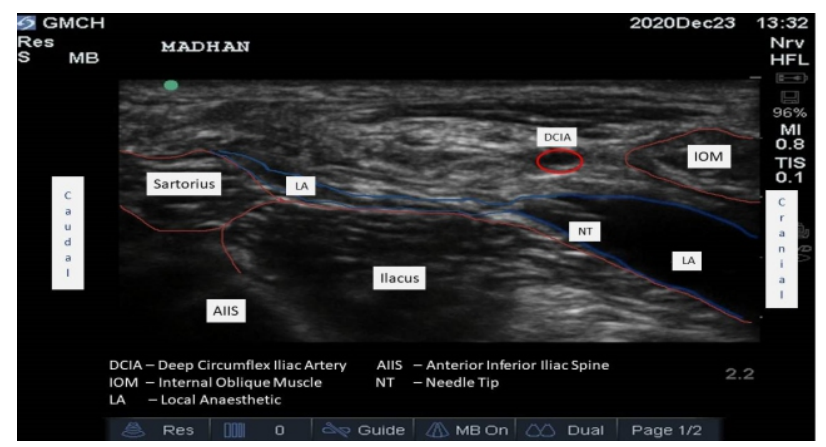

Figure 5: Supra Inguinal FICB Showing Local Anaesthetic Spread 
Advantage: When compared to infrainguinal FICB, suprainguinal FICB blocks LFCN consistently and with larger volume $\mathrm{ON}$ can also be blocked.

Block Description: Ultrasound guided suprainguinal FICB was first demonstrated by Hebbard [4] in a cadaver study. The block is performed with the patient lying supine and a linear high frequency probe $(6-13 \mathrm{MHz})$ is placed longitudinally parallel to the anterior superior iliac spine (ASIS). The probe is further moved medially till the anterior inferior iliac spine (AIIS) is reached and the fascia iliaca, sartorius, iliopsoas, and internal oblique muscles are identified (Fig. 1,2 \& 3). The deep circumflex artery can be found lying superficial to the fascia iliaca $\{$ Fig 4$\}$. At this frame the 'hour glass pattern'/'bowtie sign' formed by the iliacus muscle can be appreciated [5]. A $100 \mathrm{~mm}$ block needle is introduced caudad to cephalad direction using an in-plane approach and the needle tip is positioned beneath the fascia iliaca. Using hydro-dissection the fascia iliaca is separated from the iliacus muscle. The advised LA volume for this block is $30-40 \mathrm{ml}$. The block success depends upon the cephalic spread of LA between the ilacus muscle and the fascia where the 3 nerves lie underneath the internal oblique muscle (Fig. 5). Supra inguinal FICB can be the true 3 in 1 block as with large volume of about 40 $\mathrm{ml}$ should blockFN, ON and LFCN.

Complications: Though it is a superficial and safer block, complications like vascular injury, intramuscular/intraperitoneal injection and local anaesthetic systemic toxicity can occur.

\section{References}

1. Hebbard P, Ivanusic J, Sha S. Ultrasound-guided suprainguinal fascia iliaca block: a cadaveric evaluation of a novel approach. Anaesthesia 2011; 66:300e5.

2. Dalens B, Vanneuville G, Tanguy A. Comparison of the fascia iliaca compartment block with the 3-in-1 block in children. Anesth Analg 1989; 69: 705e13.

3. Stevens M, Harrison G, McGrail M. A modified fascia iliaca compartment block has significant morphine-sparing effect after total hip arthroplasty. Anesth Intensive Care 2007; 35: 949e52

4. Vermeylen K, Soetens F, Leunen I, Hadzic A, Van Boxtael S, Pomés J, Prats-Galino A, Van de Velde M, Neyrinck A, Sala-Blanch $X$. The effect of the volume of supra-inguinal injected solution on the spread of the injectate under the fascia iliaca: a preliminary study. J Anesth. 2018 Dec;32(6):908-913. doi: 10.1007/s00540018-2558-9. Epub 2018 Sep 24. PMID: 30250982.

5. Singh, Harsimran, Jones, David. Hourglass-Pattern Recognition Simplifies Fascia Iliaca Compartment Block. Reg Anesth Pain Med.2013;38(5):467-8. doi:10.1097/AAP.0b013e3182a1f772.

Table: Block Features

\begin{tabular}{|c|c|c|c|}
\hline Block & Advantage & limitation & Remarks \\
\hline $\mathrm{CCB}$ & Phrenic nerve sparing & Blood vessels lying in the needle path & $\begin{array}{c}\text { Excellent block for procedures from mid arm } \\
\text { and below }\end{array}$ \\
\hline SPB & $\begin{array}{l}\text { Superficial block and can be performed with the } \\
\text { patient supine }\end{array}$ & $\begin{array}{l}\text { It is not useful for posterolateral rib fractures and } \\
\text { sternal fractures. }\end{array}$ & $\begin{array}{l}\text { Safe regional analgesic option for chest trauma pain } \\
\text { management }\end{array}$ \\
\hline PENG & $\begin{array}{l}\text { Motor sparing analgesia for hip joint with consistently } \\
\text { blocking accessory obturator nerve }\end{array}$ & Deeper block so pain during needle manipulation & $\begin{array}{c}\text { Can be used as an on arrival block for hip fractures. } \\
\text { Lacks large randomized trials as of now }\end{array}$ \\
\hline SIFICB & $\begin{array}{l}\text { Blocks LFCN consistently than infrainguinal approach } \\
\text { and also proximal branches of FN }\end{array}$ & More volume of $L A$ is needed & $\begin{array}{l}\text { Useful for blocking FN, LFCN and possibly ON } \\
\text { for anaesthesia as well as analgesia }\end{array}$ \\
\hline
\end{tabular}

62 | International Journal of Regional Anaesthesia | Volume 2 | Issue 1 | January-June 2021 | Page 54-62 